\title{
Electromechanical-Traffic Model of Compression-Based Piezoelectric Energy Harvesting
}

\author{
B.C. Kok ${ }^{1}$, Saleh Gareh ${ }^{2}$, H.H. Goh ${ }^{1}$ and C. Uttraphan ${ }^{3}$ \\ ${ }^{1}$ Department of Electrical Power Engineering, Faculty of Electrical \& Electronic Engineering, UTHM 86400, Johor, Malaysia. \\ ${ }^{2} P h . D$ candidate in Electrical Engineering at Faculty of Electrical \& Electronic Engineering, UTHM 86400, Johor, Malaysia. \\ ${ }^{3}$ Department of Computer Engineering, Faculty of Electrical \& Electronic Engineering, UTHM 86400, Johor, Malaysia.
}

\begin{abstract}
Piezoelectric energy harvesting has advantages over other alternative sources due to its large power density, ease of applications, and capability to be fabricated at different scales: macro, micro, and nano. This paper presents an electromechanical-traffic model for roadway compression-based piezoelectric energy harvesting system. A twodegree-of-freedom (2-DOF) electromechanical model has been developed for the piezoelectric energy harvesting unit to define its performance in power generation under a number of external excitations on road surface. Lead Zirconate Titanate (PZT-5H) is selected as the piezoelectric material to be used in this paper due to its high Piezoelectric Charge Constant $(d)$ and Piezoelectric Voltage Constant $(g)$ values. The main source of vibration energy that has been considered in this paper is the moving vehicle on the road. The effect of various frequencies on possible generated power caused by different vibration characteristics of moving vehicle has been studied. A single unit of circle-shape Piezoelectric Cymbal Transducer (PCT) with diameter of $32 \mathrm{~mm}$ and thickness of $0.3 \mathrm{~mm}$ be able to generate about $0.12 \mathrm{~mW}$ and $13 \mathrm{~mW}$ of electric power under $4 \mathrm{~Hz}$ and $20 \mathrm{~Hz}$ of excitation, respectively. The estimated power to be generated for multiple arrays of PCT is approximately $150 \mathrm{~kW} / \mathrm{km}$. Thus, the developed electromechanical-traffic model has enormous potential to be used in estimating the macro scale of roadway power generation system.
\end{abstract}

\section{Introduction}

For the past ten years, many researchers have trained their sights on harvesting the vibration energy. This is due to its promising capacity to promote independentlypowered wireless sensors [1]. Electromagnetic [2], electrostatic [3] and piezoelectric [4], [5] are the three kinds of electromechanical transducers that have been examined as vibration-operated generators. The underlying function of these electromechanical transducers is the alteration of mechanical energy into electrical energy. The vibration and stresses generated by vehicles along public roads are among the major sources of unexploited ambient energy. The harvesting of this type of energy can be realized with the utilization of a piezoelectric transducer (PZT) [6], [7]. The generation of electric voltage by PZTs is achieved through the exploitation of loading stress and vibration.

As depicted in Fig. 1, generator effect and sensor effect are the two terms that related to the direct piezoelectric effect of the piezoelectric materials, while motor effect and actuator effect are the terms related to the converse piezoelectric effect of the piezoelectric materials. In general, direct piezoelectric effect is employed for energy harvesting. Energy harvesting is a procedure whereby energy is taken out from the environment, altered into electrical energy, and stockpiled [8].

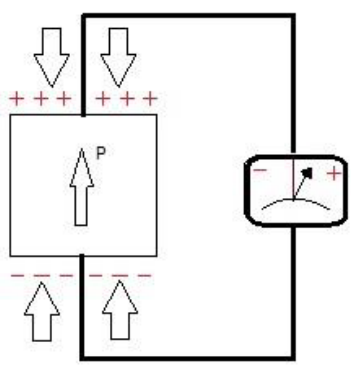

(a)

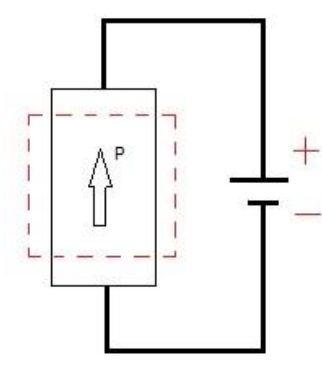

(b)
Figure 1. (a) Direct piezoelectric effect, (b) Converse piezoelectric effect.

The paper intents to present the two-degree-of-freedom (2-DOF) electromechanical model of compression-based piezoelectric harvesting unit for roadway application. A Cymbal transducer will be used to demonstrate the potential generated power and voltage of the developed model in various persuasive road vibration excitation frequencies as well as in the condition of variable external electrical loads.

\section{Related works}

The extraction of energy from vibration, temperature differentials and light are appeared to be the most effective means for micro energy harvesting. Recently, a 
compression-based energy harvester for roadways application has been introduced in [9], [10]. The harvester can be implanted into roadways for the purpose of exploiting electrical energy stemming from vibrations generated by traffic movement. Investigations revealed that a solitary harvesting module of 36 layers, under excitation of $1360 \mathrm{~N}$ of force amplitude and $6 \mathrm{~Hz}$ of frequency able to produce $85 \mathrm{~mW}$ of DC power.

A separate venture [11] employed a cantilever piezoelectric harvester to exploit the energy from vibrations produced by traffic movement over bridges. It has been observed that by maintaining a voltage between 1.8 and $3.6 \mathrm{~V}$ under a frequency lower than $15 \mathrm{~Hz}$, mean power of as much as $0.03 \mathrm{~mW}$ can be generated.

An evaluation on the effectiveness of well-accepted piezoelectric transducers used for the harvesting of energy from tarmac walkways was carried out by [12], [13]. Eventually, they came up with an energy harvesting cymbal that could harvest output power of approximately $1.2 \mathrm{~mW}$ from tarmac walkways at a vehicle load frequency of $20 \mathrm{~Hz}$. The velocity and types of the vehicle determines the frequency of the load on the implanted piezoelectric transducer. The conversion of unused acoustical energy into functional electrical energy was achieved by [14] with the development of the acoustic micro-energy harvester. At $60 \mathrm{~Hz}$ and $400 \mathrm{k} \Omega$ resistive load, the harvester successfully generated $0.9 \mathrm{~V} /\left(\mathrm{ms}^{-2}\right)$ and $1.79 \mu \mathrm{W} /\left(\mathrm{m}^{2} \mathrm{~s}^{-4}\right)$.

Table 1 gives the comparison of three research groups who working on piezoelectric roadways applications. Obviously, the recent developed technologies in piezoelectric have demonstrated the possibility to harvest huge amount of electric power from roadways [7].

Table 1. Generated energy from different research groups.

\begin{tabular}{|c|c|c|c|}
\hline Parameter & Genziko & ODOT & Innowattech \\
\hline $\begin{array}{c}\text { Generated } \\
\text { power per km } \\
\text { (single lane) }\end{array}$ & $\begin{array}{c}13-51 \\
\mathrm{MW}\end{array}$ & $486 \mathrm{~kW}$ & $100-200 \mathrm{~kW}$ \\
\hline $\begin{array}{c}\text { Vehicles per } \\
\text { hour (single } \\
\text { lane) }\end{array}$ & $\begin{array}{c}600- \\
2250\end{array}$ & 600 & 600 \\
\hline
\end{tabular}

Table 2 illustrates the approximated amount of per unit possible harvested energy from two vibration harvesting sources i.e. from human and industry.

Table 2. Generated energy from vibration sources.

\begin{tabular}{|c|c|}
\hline $\begin{array}{c}\text { Source of energy } \\
\text { (Vibration/ motion) }\end{array}$ & Harvested power \\
\hline Human & $4 \mu \mathrm{Wcm}^{-2}$ \\
\hline Industry & $100 \mu \mathrm{Wcm}^{-2}$ \\
\hline
\end{tabular}

\section{Methodology}

A two-degree-of-freedom (2-DOF) electromechanical model of the piezoelectric harvesting unit under the exciting force is portrayed in Fig. 2. This piezoelectric model receives its input from the force produced by traffic movement. This force is denoted in the model as $F(t)$.

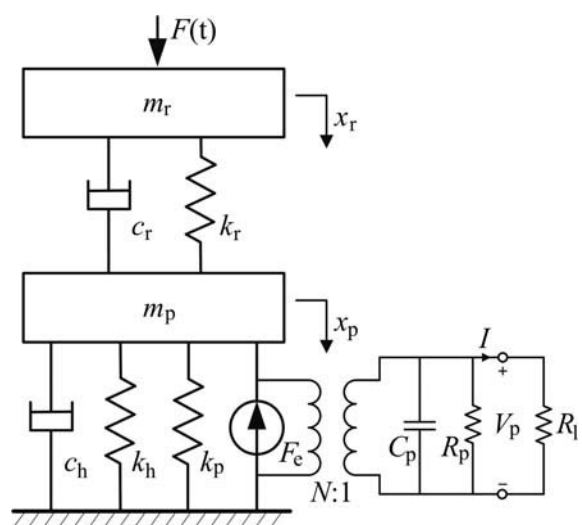

Figure 2. The electromechanical model of piezoelectric energy harvesting [10]

The symbols represented in the electromechanical model as well as the following equations are defined as follows:

$m_{\mathrm{r}}, c_{\mathrm{r}}$ and $k$ are the mass, damping and elastic coefficient of the rubber layer, respectively.

$m_{p}$ and $k_{\mathrm{p}}$ are the mass and elastic coefficients of the piezoelectric stack, correspondingly.

The mechanized damping and elastic coefficients of the harvester's mechanical structure are symbolized as the parameters of $c_{h}$ and $k_{h}$, respectively.

The deformation of the rubber layer and piezoelectric wafer-stack attributed to the external force are symbolized as the parameters $X_{r}$ and $X_{p}$, respectively.

The clamped capacitance of the piezoelectric wafer-stack is symbolized as $C_{p}$

The piezoelectric leakage resistance and the electric loss property of the piezoelectric wafer-stack are symbolized as $R_{p}$ and $R_{l}$, respectively.

The angular frequency of the exciting vibration is symbolized as $\omega$ and the frequency equivalent fuction of $F(t)$ is symbolized as $F(\omega)$.

The external electrical load is symbolized as $R$.

The normalized frequencies are symbolized as $\alpha$ and $\varphi$. The normalized electrical resistance is symbolized as $\beta$, and the electromechanical coupling alternative is symbolized as $k_{e m}$.

The interrelation parameters that can be computed are given as follows:

$$
\begin{gathered}
\omega_{1}=\sqrt{\frac{k_{r}}{m_{r}}}, \omega_{2}=\sqrt{\frac{k_{a}}{m_{p}}} \\
\xi_{1}=\frac{c_{r}}{2 \sqrt{m_{r} k_{r}}}, \xi_{2}=\frac{c_{h}}{2 \sqrt{m_{p} k_{a}}} \\
\mu=\frac{m_{r}}{m_{p}} \\
k_{a}=k_{p}+k_{h} \\
\alpha=\frac{\omega_{1}}{\omega_{2}}
\end{gathered}
$$




$$
\begin{gathered}
\varphi=\frac{\omega}{\omega_{2}} \\
\beta=\omega_{2} R C_{p} \\
k_{e m}^{2}=\frac{N^{2}}{C_{p} k_{a}}
\end{gathered}
$$

The generated voltage from the harvester is defined as:

$$
V=\frac{\left.F(\omega) k_{e m}^{2} \beta \varphi \alpha\right)}{N\left(A_{1}^{2}+A_{2}^{2}\right)}\left[\left(A_{1} \alpha+2 A_{2} \xi_{1} \varphi\right)+\left(2 A_{1} \xi_{1} \varphi-A_{2} \alpha\right) j\right]
$$

The generated electric power by the harvester is written as:

$$
P=\frac{\left.F(\omega)^{2} k_{e m}^{4} \beta^{2} \varphi^{2} \alpha^{2}\right)\left\{\left(A_{1} \alpha+2 A_{2} \xi_{1} \varphi\right)^{2}+\left(2 A_{1} \xi_{1} \varphi-A_{2} \alpha\right)^{2}\right\}}{2 N^{2}\left(A_{1}^{2}+A_{2}^{2}\right) R}
$$

where,

$$
\begin{gathered}
A_{1}=A_{3} \beta \varphi+\left(\alpha^{2}-\varphi^{2}\right) \beta k_{e m}^{2} \varphi+A_{4} \\
A_{2}=A_{4} \beta \varphi+2 \xi_{1} \alpha \beta k_{e m}^{2} \varphi^{2}-A_{3} \\
A_{3}=\alpha^{2}-\varphi^{2}-(1+\mu) \alpha^{2} \varphi^{2}+\varphi^{4}-4 \xi_{1} \xi_{2} \varphi^{2} \alpha \\
A_{4}=2 \varphi\left[\alpha\left(1-\varphi^{2}-\mu \varphi^{2}\right) \xi_{1}+\left(\alpha^{2}-\varphi^{2}\right) \xi_{2}\right]
\end{gathered}
$$

The electromechanical model is derived based on the compression-based piezoelectric cymbal transducer [13]. This transducer enables the endurance of hefty loads present in the domain of public infrastructure schemes. A harvester equipped with this transducer can be implanted into public buildings, roads and highways for electric energy harvesting. The common properties for the piezoceramics are given in Table 3.

Table 3. The piezoelectric material properties.

\begin{tabular}{|c|c|c|c|}
\hline Property & PZT-5H & PZT-8 & PZT-5A \\
\hline$d_{33}[\mathrm{pm} / V]$ & 650 & 225 & 390 \\
\hline$d_{33}^{\text {eff }}[\mathrm{nm} / V]$ & 110 & 38 & 66 \\
\hline$\varepsilon_{33}^{T} / \varepsilon_{0}$ & 3800 & 1000 & 1800 \\
\hline$C_{p}[n F]$ & 1745 & 459 & 826 \\
\hline
\end{tabular}

The $d$-coefficient is the proportionality constant between dielectric displacement and stress or strain and electric field strength and their relationships are given in Equations (3) and (4). High $d$-coefficient is desirable in materials utilized as actuators, such as in motional and vibrational applications.

$$
\begin{aligned}
& D=\varepsilon^{T} E+d T \\
& S=s^{E} T+d T
\end{aligned}
$$

where,

$D$ is the dielectric displacement, $\varepsilon$ is the permittivity,

$T$ is the stress,

$E$ is the electric field strength,

$S$ is the strain, $s$ is the elastic compliance,

$d$ is the piezoelectric constant.

Lead Zirconate Titanate (PZT) is the most favored material in the field of ceramic engineering in which it comes in a variety of forms.

It is frequently used in the application of transducers, sensors and actuators. Presently, the most frequently utilized in engineeringfield of piezoceramics are PZT-5A and PZT-5H [15]. The piezoelectric model used in this study is PZT-5H which is a soft ceramic material that comes with a greater piezoelectric coefficient (direct charge coefficient), $d_{33}$ as well as larger dielectric permittivity, $\varepsilon_{33}^{T}$.

A compression-based piezoelectric model is proposed to be utilized in the roadway energy harvester. Under the dynamic forces of compression, every single compression cycle produces energy in the form of pulses [10]. The model revealed that under harmonic excitation, the recommended stack harvester of 36 layers can produce as much as $200 \mathrm{~mW}$ of electrical energy [9]. This level of electrical energy is enough for the operation of nearly all forms of wireless sensors. Fig. 3 displays the electricity harvesting during the passage of a car (or any other vehicle) across the harvesters implanted in the roadway.

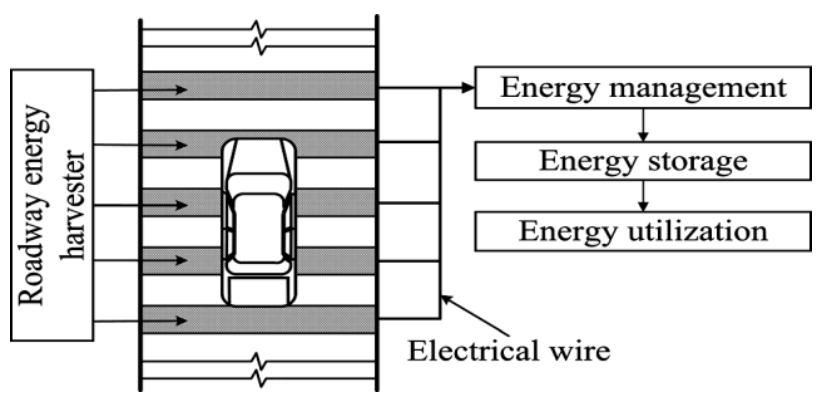

Figure 3. Roadway energy harvester on the road [9].

In order to demonstrate the potential of energy harvesting, a piezoelectric harvester unit is simulated so that the movement of a vehicle on the roadway is exploited. The electromechanical-traffic model is developed in Matlab environment in which the single cell of the piezoelectric consist of a poled piezoelectric disk (fully electrodes on top and bottom surface) which is sandwich with both truncated cone-shaped metal end caps, each containing a shallow air-filled cavity on their inner surface as shown in Fig. 4.

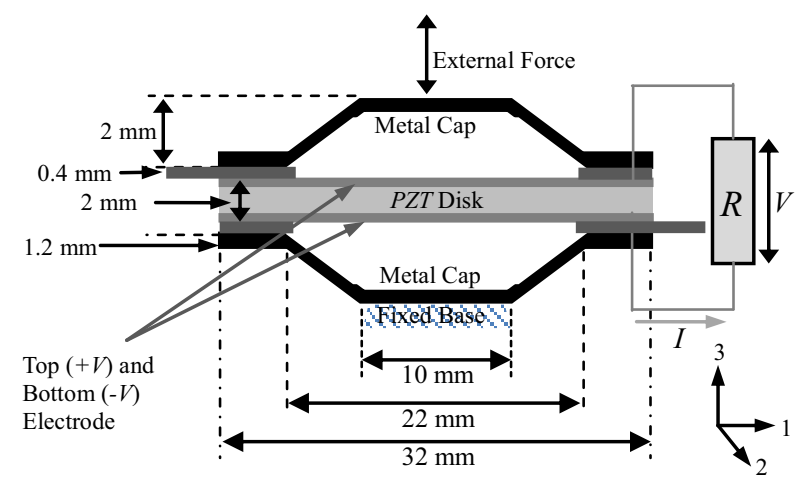

Figure 4. Piezoelectric Cymbal Transducer (PCT). 


\section{Simulation results}

The passage of a vehicle across a pavement produces minor vibrations in the pavement. The conversion of these vibrations into electricity is achievable through the implanting of piezoelectric sensors into the road. The vibrations in fact depend on the road condition and type of vehicle passing through in terms of speed and mass of the vehicle.

The relationships between the amplitudes of the generated power and voltage with varying external electrical loads under the vibration excitation of 4 and 20 $\mathrm{Hz}$, respectively is displayed in Figures 5 and 6.
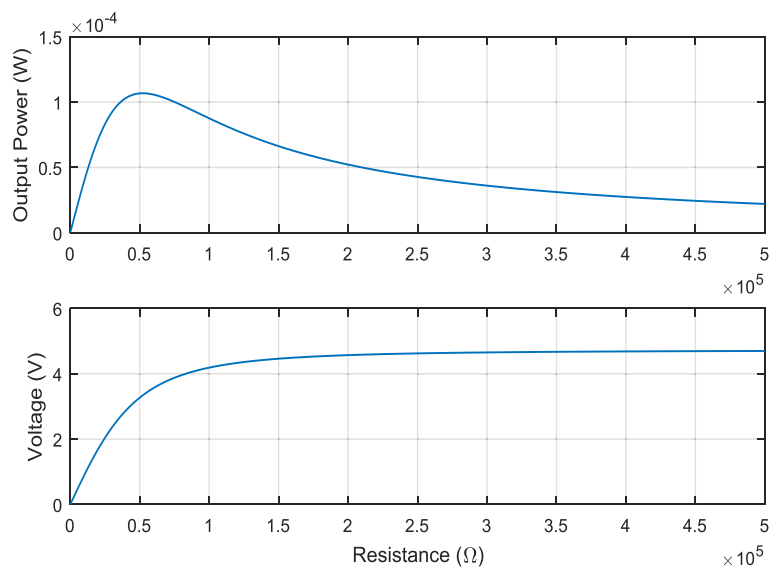

Figure 5. Output power and voltage vs. electrical loads under vibration excitation of $4 \mathrm{~Hz}$.
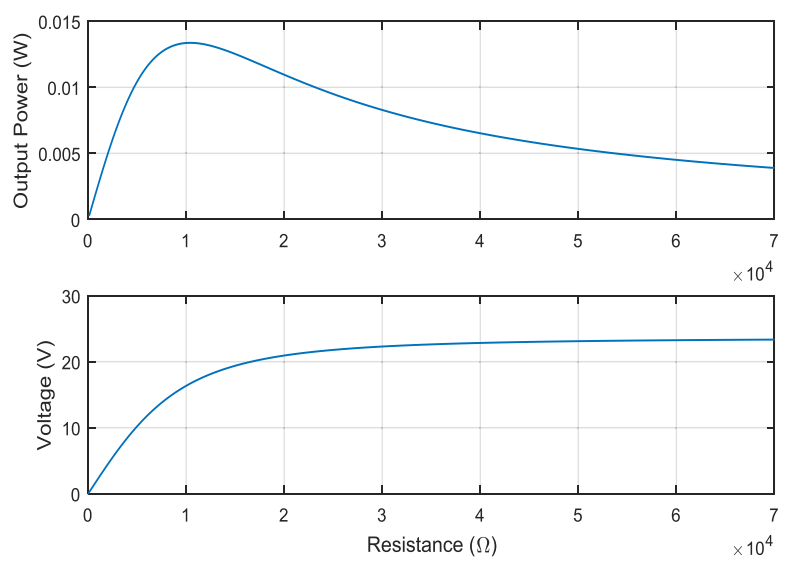

Figure 6. Output power and voltage vs. electrical loads under vibration excitation of $20 \mathrm{~Hz}$.

The highest amplitude of output power as recorded in Fig. 5 with the vibration excitation of $4 \mathrm{~Hz}$ is $0.12 \mathrm{~mW}$ with an external load resistance of $50 \mathrm{k} \Omega$. Within a particular external resistance span, the voltage is raised together with the value of the external loads. Upon the external resistance surpassing a particular value, the voltage becomes constant. The maximum voltage level is remained constant at about $4.8 \mathrm{~V}$ with the external load resistance of $200 \mathrm{k} \Omega$ onwards. Fig. 6 exhibits a significant maximum voltage increased to about $23 \mathrm{~V}$ with the vibration excitation of $20 \mathrm{~Hz}$. Likewise, the output power is raised to about $13 \mathrm{~mW}$ at $10 \mathrm{k} \Omega$ of load.

The increase of excitation frequency leads to the raise of harvested power as well as the voltage as shown in Fig. 7 .
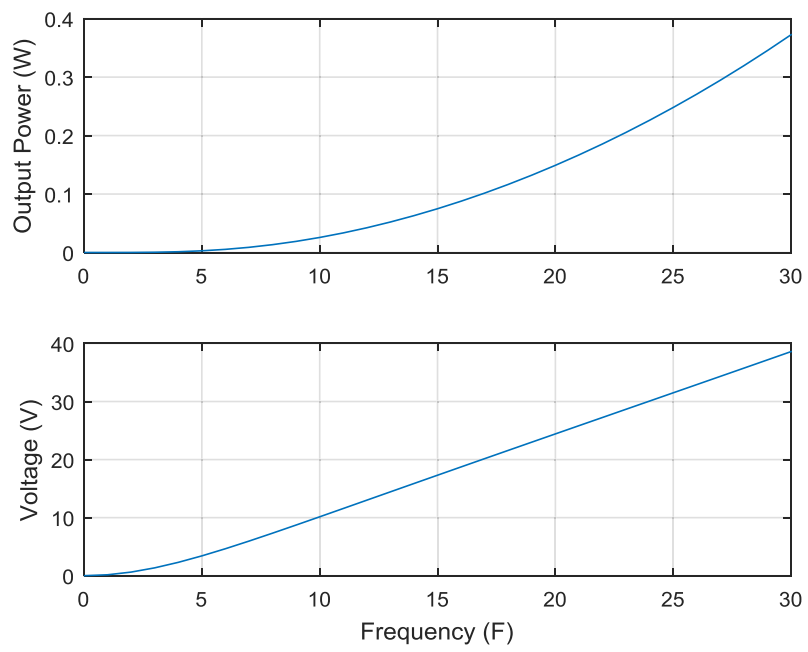

Figure 7. Harvested power and voltage vs. frequency.

Fig. 8 demonstrates the utilization of Cellular Automata $(\mathrm{CA})$ to realize the simulation results of harvested power and voltage in real-time for a single lane traffic model.

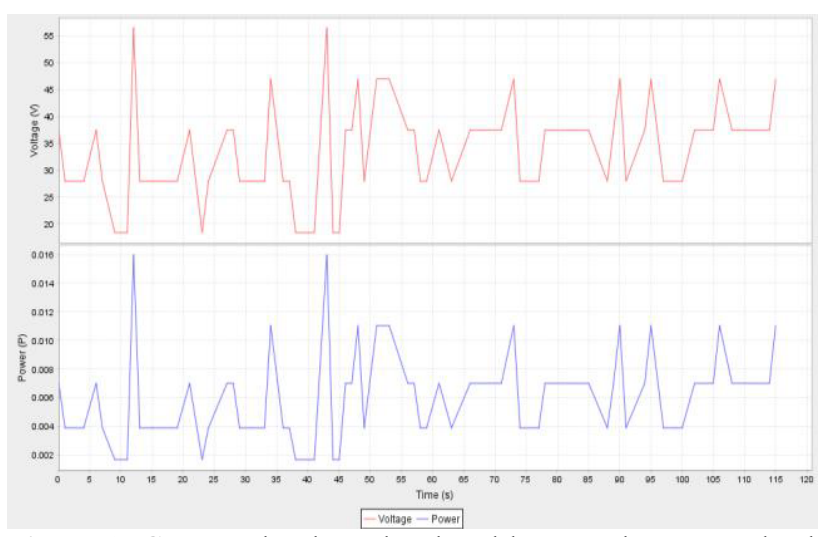

Figure 8. Generated voltage level and harvested power at load resistance of $100 \mathrm{k} \Omega$.

The passage of 70 two-axle vehicles with an average speed of $70 \mathrm{~km} / \mathrm{h}$ within 2 minutes across a single PCT resulted in an estimated overall power generation of 417 $\mathrm{mW}$ and average voltage of $34 \mathrm{~V}$. The PCT arrays can be placed along one kilometer stretch of road to elevate the amount of power harvested.

\section{Conclusion}

This investigation delved into energy harvesting with a compression-based piezoelectric harvester that exploits vibrations derived from traffic movement. The energy acquired through this process can be used to supply electricity for the operation of remote control tools utilized in transportation systems. The outcomes from simulation revealed that the generation of as much as 13 $\mathrm{mW}$ of power under the excitation frequency of $20 \mathrm{~Hz}$ is achievable with a single PCT harvester. Other than the piezoelectric harvester, the external electrical load resistance also plays a role in determining the level of electrical power realized through compression-based piezoelectric harvesting system. 
As an elevated velocity is indicative of an elevated exciting frequency, the output power rises in tandem with the velocity of a passing vehicle. With several series' of PCT, it is well within the means of the recommended roadway harvester to harvest about $150 \mathrm{~kW}$ per kilometer from a highway with a traffic volume of 600 vehicles per hour. The amount of power harvested is sufficient for the powering of common roadway appliances that include lighting schemes, emergency communication equipment and roadside billboards.

\section{Acknowledgement}

The authors gratefully acknowledged the financial support by the Ministry of Higher Education (MOHE) Malaysia under ERGS research grant with the vote number of E035 for this research works. The authors wish to express their heartfelt appreciativeness to Office for Research, Innovation, Commercialization and Consultancy Management (ORICC), UTHM for their continuous support and assistance in managing the research grant.

\section{References}

1. H.Y. Wang, , L.H. Tang, Y. Guo, X.B. Shan, and T. Xie, Journal of Zhejiang University SCIENCE A, 15, 711-722 (2014)

2. M. El-Hami, P. Glynne-Jones, N.M. White, M. Hill, S. Beeby, E. James, A.D. Brown, and J.N. Ross, Sensors and Actuators A: Physical, 92, 335-342 (2001)

3. M. Miyazaki, , H. Tanaka, G. Ono, T. Nagano, N. Ohkubo, T. Kawahara, and K. Yano, Low Power Electronics and Design, 2003. ISLPED'03.
Proceedings of the 2003 International Symposium on, 193-198 (2003)

4. C. Keawboonchuay and T.G. Engel, Pulsed Power Conference, 2003. Digest of Technical Papers. PPC2003. 14th IEEE International, 1, 327-330 (2003)

5. J. Yang, Z. Chen, and Y. Hu, Ultrasonics, Ferroelectrics, and Frequency Control, IEEE Transactions on, 54, 190-195 (2007)

6. S.F. Ali, M.I. Friswell, and S. Adhikari, Journal of Intelligent Material Systems and Structures, 1929 (2011)

7. D. Hill, A. Agarwal and N. Tong, Assessment of Piezoelectric Materials for Roadway Energy Harvesting : Cost of Energy and Demonstration Roadmap: Final Project Report, (2014)

8. S. Vassiliadis, Advances in Modern Woven Fabrics Technology, (2011)

9. X. Jiang, Y. Li, J. Li, J. Wang, and J. Yao, Journal of Renewable and Sustainable Energy, 6, 043110 (2014)

10. X. Jiang, Y. Li, J. Wang, and J. Li, International Journal of Smart and Nano Materials, 5, 152-168 (2014)

11. M. Peigney and D. Siegert, Smart Materials and Structures, 22, 095019 (2013)

12. H. Zhao, J. Ling, and J. Yu, Journal of the Ceramic Society of Japan, 120, 317-323 (2012)

13. H.G. Chua, B.C. Kok, and H.H. Goh, Energy and Sustainability V, 186, 103 (2014)

14. K.F. Chen, J.H. Ho, and E.H. Yap, World Academy of Science, Engineering and Technology, International Journal of Electrical, Computer, Energetic, Electronic and Communication Engineering, 9, 708-714 (2015)

15. K.A. Cook-Chennault, N. Thambi, and A.M. Sastry, Smart Materials and Structures, 17, 043001 (2008) 